\title{
Projection-Based Metal-Artifact Reduction for Industrial 3D X-ray Computed Tomography
}

\author{
Artem Amirkhanov, Christoph Heinzl, Michael Reiter, Johann Kastner, and M. Eduard Gröller, Member, IEEE
}
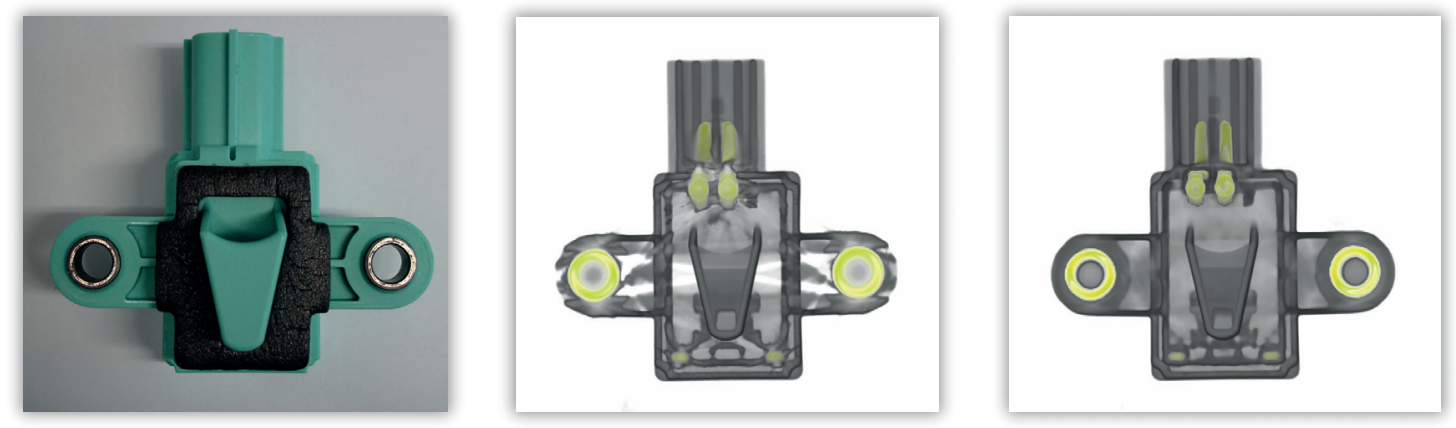

Fig. 1. The plug specimen photo (left) and volume renderings before (center) and after (right) metal-artifact reduction.

\begin{abstract}
Multi-material components, which contain metal parts surrounded by plastic materials, are highly interesting for inspection using industrial 3D X-ray computed tomography (3DXCT). Examples of this application scenario are connectors or housings with metal inlays in the electronic or automotive industry. A major problem of this type of components is the presence of metal, which causes streaking artifacts and distorts the surrounding media in the reconstructed volume. Streaking artifacts and dark-band artifacts around metal components significantly influence the material characterization (especially for the plastic components). In specific cases these artifacts even prevent a further analysis. Due to the nature and the different characteristics of artifacts, the development of an efficient artifact-reduction technique in reconstruction-space is rather complicated. In this paper we present a projection-space pipeline for metal-artifacts reduction. The proposed technique first segments the metal in the spatial domain of the reconstructed volume in order to separate it from the other materials. Then metal parts are forward-projected on the set of projections in a way that metal-projection regions are treated as voids. Subsequently the voids, which are left by the removed metal, are interpolated in the 2D projections. Finally, the metal is inserted back into the reconstructed 3D volume during the fusion stage. We present a visual analysis tool, allowing for interactive parameter estimation of the metal segmentation. The results of the proposed artifact-reduction technique are demonstrated on a test part as well as on real world components. For these specimens we achieve a significant reduction of metal artifacts, allowing an enhanced material characterization.
\end{abstract}

Index Terms-Metal-artifact reduction, multi-material components, visual analysis, 3D X-ray computed tomography.

\section{INTRODUCTION}

Three-dimensional X-ray computed tomography (3DXCT) is a powerful technique for generating a digital $3 \mathrm{D}$ volumetric dataset of a specimen from 2D X-ray penetration images (projections). The main advantage of 3DXCT is its ability to capture both the interior and the exterior structures of a specimen including a detailed material characterization in one single scan. Having been used in medical diagnostics

- Artem Amirkhanov is with the Institute of Computer Graphics and Algorithms, Vienna University of Technology, E-mail: artem@cg.tuwien.ac.at.

- Christoph Heinzl is with the Upper Austrian University of Applied Sciences, Wels Campus, E-mail: Christoph.Heinzl@fh-wels.at.

- Michael Reiter is with the Upper Austrian University of Applied Sciences, Wels Campus.

- Johann Kastner is with the Upper Austrian University of Applied Sciences, Wels Campus.

- Eduard M. Gröller is with the Institute of Computer Graphics and Algorithms, Vienna University of Technology, E-mail: groeller@cg.tuwien.ac.at.

Manuscript received 31 March 2011; accepted 1 August 2011; posted online 23 October 2011; mailed on 14 October 2011.

For information on obtaining reprints of this article, please send emailto:tvcg@computer.org. for years, 3DXCT is increasingly employed in industry as a method for non-destructive testing and quality control. Especially in the area of metrology, 3DXCT is increasingly applied due to its advantageous characteristics. As compared to methods of conventional metrology, $\mathrm{CT}$ is the only method which facilitates dimensional measurements of the internal and inaccessible structures of a component.

Especially this property makes 3DXCT highly convenient for the inspection of multi-material components (MMCs). MMCs are specimens which are manufactured from different materials with high density variation, e.g., plastic-metal components or composite materials with metal inlays. For MMCs one important goal of data analysis is material characterization. Another key goal is interface detection to measure features of interest in each of the materials. A high dynamic range of the attenuation values in the scan data causes a variety of artifacts and complicates the characterization of component and material. Artifacts are artificial structures in the reconstructed dataset, which do not correspond to structures of the measured specimen [6]. The presence of artifacts generates distortions in the resulting volume dataset. They show up as greyvalue variations and deviations in the reconstructed dataset. Some of the most common artifact types of 3DXCT are noise-induced streaks, beam hardening, partial volume effects, aliasing, and scattered radiation [10]. In severe cases the bad quality of the resulting data might prevent any further exploration and analysis. 


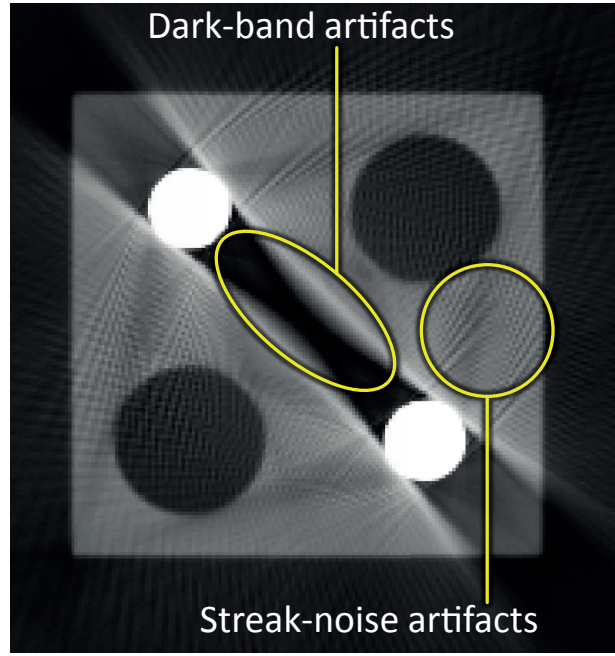

Fig. 2. Example of metal artifacts. Dark-band and streak-noise artifacts are marked.

For polychromatic radiation as used in 3DXCT the correlation between attenuation and penetration length is nonlinear. The polychromatic spectrum of an X-ray beam is hardened as it traverses through the material. This means that higher energy photons pass through the material, while lower energy photons are absorbed. What remains is a modified (hardened) spectrum containing mainly the higher energy portions [6]. This effect is called beam-hardening. Beam-hardening causes two types of artifacts: cupping artifacts and streaking artifacts (bright or dark bands or streaks between dense objects in the image) [1]. Streaking artifacts are commonly known as metal artifacts since they usually appear when the low-absorbing material of a specimen contains metal inclusions such as pins, screws or nails. Therefore, metal artifacts are most common when MMCs are investigated using 3DXCT. Metal artifacts appear as massive bright or dark bands between the highly absorbing components. They also appear as high frequency streak-noise originating at the boundary of metal inclusions. An example of metal artifacts causing distortions of the media between the metal pins and high frequency streak noise is given in Figure 2. Due to these artifacts material characterization and dimensional measurement is hindered and in several regions impossible.

The problem of metal artifacts in MMCs is of high importance and prevalent in industrial X-ray computed tomography (XCT). MMCs are very common as most modern industrial parts have multi-material components, at least after assembly. There is a big variety of materials and the range of attenuation coefficients is in many cases very high as compared to medical XCT. Using high energies in industrial XCT scanning typically introduces blurring of low-absorbing materials and, therefore, does not allow one to efficiently capture high-frequency details.

In this paper we adopt a projection-based workflow for metalartifacts reduction (MAR) for industrial XCT. We apply this pipeline to MMCs containing three materials: air, plastic and metal. The motivation behind this workflow is as follows: metal artifacts result from those areas in the projections where metal is represented. So, if we find the areas representing metal and substitute the data with some reasonable approximations, we will be able to reduce artifacts in the resulting reconstructed volume. Since the metal parts are blanked out we will get an artifact-reduced dataset but still without the metal components. Subsequently, we then insert metal data back into the resulting volume dataset. An overview of the metal-artifact reduction-workflow is presented in Figure 3. The detailed description of the workflow is given in Section 3.

The given workflow is semi-automatic. Most of the steps (e.g., reconstruction, forward-projection and interpolation) can be done automatically. On the other hand, the material separation requires setting

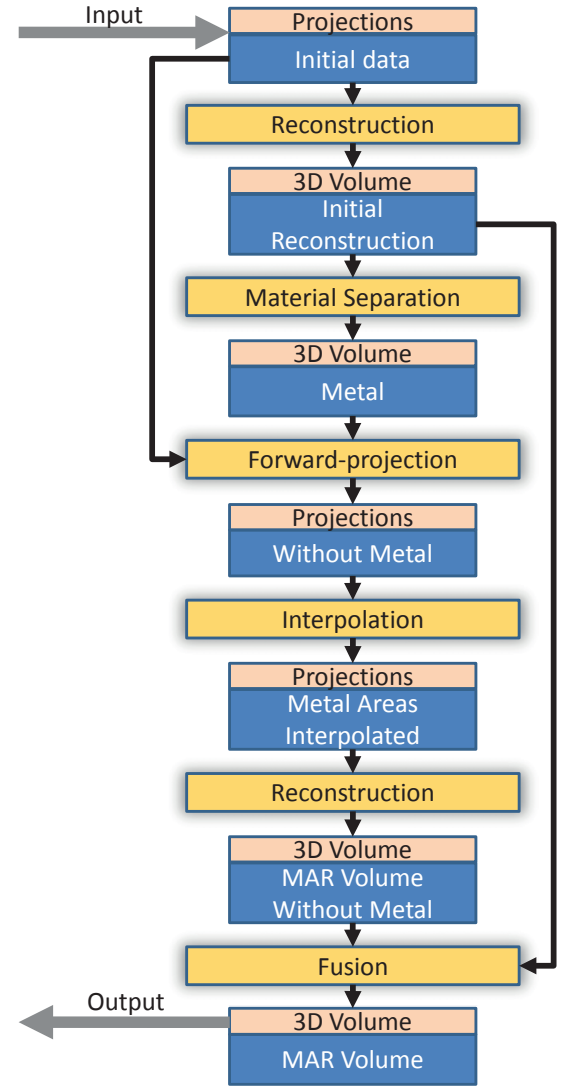

Fig. 3. Projection-based metal-artifact reduction workflow.

an attenuation threshold by the user.

We present a visual tool integrating all steps of the workflow. It provides the user with a setup-wizard like interface, guiding him through the steps and allowing the specification of all necessary parameters. After all the parameters are estimated, the workflow is executed for the specified datasets and the result of MAR is shown to the user. Comparative visualisation is provided to compare the volume before and after MAR, and explore the introduced differences. The parameter specification is guided using a set of visual analysis techniques. They allow one to estimate the impact of the parameters on the MAR result and provide various visualizations for decision support. Basic concepts and the visual analysis functionality of the tool are described in Section 4 .

The main contributions of this paper are:

- Adaptation of a projection-based MAR workflow for 3DXCT (and in particular for industrial 3DXCT)

- Integration of the workflow within one tool. It visually guides the user through the workflow pipeline and provides the visualanalysis functionality for an interactive visual parameter estimation.

\section{Related Work}

The problem of beam hardening in general and metal artifacts in particular is important and prevalent for both medical and industrial computed tomography. There are several groups of approaches which deal with this problem.

The reduction of metal artifacts in the spatial domain of the reconstructed volume is a highly challenging task. Due to the physical causes of metal artifacts, there is no explicit criterion which would allow the detection of the most artifact-affected areas in order to perform the necessary corrections. One possibility is to use dual-energy approaches. Two scans of the MMC specimen are done with different 
energies. The low-energy scan achieves a better resolution due to a smaller focal spot of the X-ray source, capturing more high-frequency details but strongly suffering from the metal artifacts. The high-energy scan on the other hand is far less affected by metal artifacts but has a lower resolution due to a bigger focal spot size. By fusing two volumes obtained from the low energy and high energy scan one can get a better representation which has both a good resolution and less metal artifacts. Heinzl et al. [7] propose a custom multi-scan fusion scheme which locally fuses the complete datasets of scans using different energies. Additionally there are dual-energy CT (DECT) approaches which calculate the real density and the effective atomic number out of the greyvalues of both datasets [21].

Another way to deal with metal artifacts is to use projection-based techniques. In this case metal artifacts are reduced by improving 2D projection images obtained directly from the scanning device. Projections are processed in a way to reduce metal artifacts in the resulting $3 \mathrm{D}$ volume. The reconstruction algorithm is then applied to the corrected projections resulting in a $3 \mathrm{D}$ volume with reduced metal artifacts. Various metal-artifact reduction techniques using the projection (raw) data are deployed in the area of industrial XCT.

For single material components there are several methods to compensate for artifacts applying a linearization technique for the greyvalues. The Iterative Artifact Reduction (IAR) method by Kasperl [15] is an iterative multistage process. The IAR is based on the linearization technique of Herman [8]. It applies a nonlinear characteristic correction curve on the projection data. The curve is extracted from the volume at every step of the reconstruction. In each iteration the curve is enhanced and the artifacts in the dataset are reduced. Hopkins et al. [9] introduced a related method. The corrections are based on the calculation of the attenuation and scattering processes characteristic of an object composed of a single material. The beam-hardening corrections are applied directly to the projection data prior to reconstruction. The corrections are derived using object geometry extracted from the initial reconstruction. The main disadvantage of the IAR method is based on the assumption that the specimen consists of one specific material or material mixture. MAR methods for single material objects are not suitable in the case when MMCs are used. Nevertheless a big portion of industrial components are MMCs, at least after assembly. In this application area of industrial 3DXCT the most severe artifacts are introduced. This prevents reliable dimensional measurements and material exploration (e.g., determining the porosity of the plastic). Currently, to avoid artifacts due to MMCs, a disassembly into single material components and subsequent separate scans are carried out. This requires the modification and in many cases the destruction of the scanned object. Furthermore, scanning time and memory consumption are increased proportionally to the number of materials in the MMC.

A multi-material correction method by Krumm et al. [17] expands the IAR method for MMCs. Materials are segmented in the reconstructed CT image. Then mono-energetic and poly-energetic reprojections are calculated using ray casting. The difference between both reprojections is used as the amount of correction for the initial projections. The whole correction process is iterative. One important limitation of the iterative MAR methods is a high computational cost which results in long processing times. In many cases such a performance is not suitable for 3DXCT where high-resolution 3D data are used. Xue et al. [33] do metal segmentation through a custom region growing directly in the sinogram of the 2D DECT data. However, segmentation in the sinogram does not depend on the actual material density and works well only for a limited list of cases.

Several approaches are used in medical $C T$ for removing artifacts caused by metal implants such as tooth implants and dental fillings, prosthetic devices, surgical clips and electrodes. The artifacts arising in the medical XCT images affect diagnosis and treatment planning and therefore seriously limit the clinical value of the XCT scan. One of the first works on metal artifact reduction in medical XCT was by Kalender et al. [14]. It proposed segmentation of the metal implant in the reconstructed 2D slice image. A semi-automatic segmentation is considered. First the operator approximately delineates the metal, then an exact determination of the metal boundaries is done. Forward projection is used to find the metal areas in the projections. Subsequently linear interpolation is used to substitute the data in those regions where metal projects to. Finally, a filtered back-projection creates an image with reduced artifacts. Subsequent work by Yu et al. [34] does the metal segmentation directly in the projections. Veldkamp et al. [30] applies a complex segmentation strategy in projection space. Three interpolation methods are investigated. Only subtle differences between interpolation methods were found. Oehler and Buzug [20] find a directional interpolation scheme following the flow of the projection data to be superior to linear and polynomial interpolation methods. Additionally, an iterative reconstruction algorithm is used for better artifact reduction. Different methods are used in order to reduce metal artifacts through image inpainting instead of interpolation. Duan et al. [3] achieve a good approximation using TV-inpainting [26]. Other image inpainting techniques can be used as well for this purpose [2, 25]. Again, high computational cost and memory requirements of image inpainting algorithms make them less applicable to large 3DXCT data.

In the area of medical XCT, metal implants in itself are of lower clinical interest as opposed to the surrounding tissues. We are not aware of any works which apply similar metal-artifact reduction in medical cases when metal parts are of interest (e.g., dental fillings, projectiles etc.). In the presented works metal parts are excluded from the final scan image or are only approximately denoted. On the contrary, in industrial XCT metal parts of the MMCs are of high interest as they have to be explored and measured along with other materials. The workflow presented in this paper allows the insertion of the metal parts back into the volume with reduced artifacts. In this way the resulting volume contains all the materials of the MMC specimen which makes it possible to explore the metal parts and their interface with other materials.

All mentioned related work in medical XCT works with 2D slice images but not $3 \mathrm{D}$ volumes. In this paper we propose a technique which is capable of reducing metal artifacts for 3D volumes resulting from a 3DXCT scan. This imposes certain restrictions on the performance of every individual step of the workflow but allows processing the MAR for the dataset on the order of several minutes. At the end of the related work section we will mention works from the adjacent research areas which are closely related to the presented method.

Common simulation approaches such as Monte-Carlo simulations [13, 23], hybrid approaches [18, 28] and discrete simulations [22] of XCT are used to predict the results of real measurements by computing the interaction of virtual X-rays with a digital model.

Fast 3DXCT reconstruction using the GPU is used in order to achieve an improved performance compared to the CPU [31]. Using modern graphics hardware $\mathrm{Xu}$ and Mueller achieve real-time 3DXCT reconstuction [32].

Visualization of industrial 3DXCT data is an important area of scientific visualization. Hadwiger et al. [5] explore volume data for detection and classification of features such as pores based on certain parameters (e.g., size, time, density). Huang et al. [11] explore various visualization techniques for nondestructive testing applications.

\section{Projection-Based Metal-Artifacts Reduction}

As input data, the pipeline uses $2 \mathrm{D}$ projections of the specimen obtained from the scanning device. A 3D volume is generated from the projection images using the filtered back-projection algorithm by Feldkamp et al. [4] (Section 3.1). In the material separation stage, the metal is segmented in the reconstructed volume and separated from the other materials (Section 3.2). In this stage we find the voxels which contain the metal. The resulting intermediate volume dataset consists only of metal parts. Next, we map the metal-containing voxels from the reconstructed volume to the initial set of projection images using forward-projection (Section 3.3). The areas of metal are blanked out. The next step of the workflow is the interpolation of the voids left by the metal components in the set of $2 \mathrm{D}$ projections (Section 3.4). As a result of this stage we have a set of projections containing plastic and voids filled with the interpolated data. Then, the reconstruction algorithm is applied to the set of projections containing plastic and 


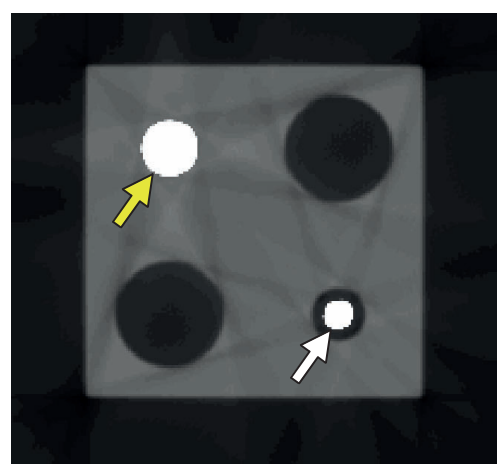

(a)

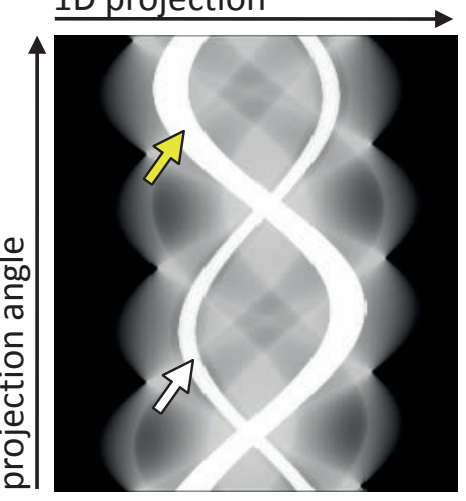

(b)

Fig. 4. Example of a 2D sinogram: (a) a scanned object (here in 2D); (b) a sinogram of 720 1D projections of this image. The arrows on the left image indicate the cross-section of two metal pins (bright white areas). The arrows on the right image indicate the trajectories of the metal pin projections (bright white sinusoidal tracks).

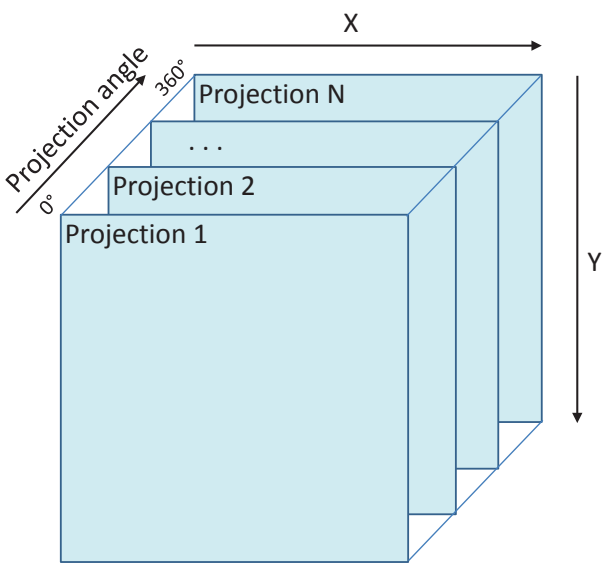

Fig. 5. 3D sinogram data representation.

interpolated data. This produces a 3D volume with plastic which is almost free from metal artifacts. Finally, data fusion is done in order to combine the two intermediate volumes: the volume containing only metal parts and the volume containing metal-artifacts free plastic (Section 3.5). The metal is inserted back into the volume reconstructed from the interpolated projections during the fusion stage.

Our workflow processes $2 \mathrm{D}$ projection images. The projection images are obtained either from scanning or from a corresponding simulation. Every projection is stored in a $2 \mathrm{D}$ image. It represents the attenuated X-rays which are partially absorbed by the scanned specimen and recorded by the detector. The projection direction is given by a projection angle. A complete set of projection images is obtained by rotating the specimen stepwise on a rotary plate. This corresponds to a $360^{\circ}$ stepwise variation of the projection direction around the specimen. In 2DXCT, the projection data is often represented as a sinogram, which is simply the 2D array of data containing the complete set of ordered $1 \mathrm{D}$ projections obtained using line scanners. In this way the projection angle is treated as the second dimension in the sinogram, besides the detector pixels of the line scanner. The resulting sinogram representation reflects inter-projection connections. For example, all projections of a point in the spatial domain will appear as a sinusoid in the sinogram. An example of a $2 \mathrm{D}$ sinogram of $1 \mathrm{D}$ projections is given in Figure 4. For 3DXCT we treat the set of 2D projections as a $3 \mathrm{D}$ sinogram, which is a $3 \mathrm{D}$ volume containing the stack of all $2 \mathrm{D}$ projections (see Figure 5). An example of a 3D sinogram is given in Figure 6. On the left the specimen is shown in the spatial domain and on the right the 3D sinogram is depicted. Three corresponding metal elements are marked with colored arrows. The areas where no metal is projected to are removed from the sinogram for illustration purposes.

The vertical amplitude of this curve is zero if the point has no offset from the X-ray source along the rotation axis and increases with a greater offset. Due to the vertical component of the curves, the decomposition of a $3 \mathrm{D}$ sinogram into a set of $2 \mathrm{D}$ sinograms representing data slices perpendicular to the rotation axis is not possible for conebeam CT. This is in contrast to parallel-beam CT. With a cone-beam $\mathrm{CT}$ only the mid-plane can be represented as a 2D sinogram. All steps of the workflow that operate on projection images use 3D sinogram data representation.

In order to get the final artifact-reduced 3D volume, the user has to go through all the steps of the workflow. Every step takes either a set of $2 \mathrm{D}$ projection images or a $3 \mathrm{D}$ volume as input and likewise produces such data as output. The user is visually guided through the workflow. It is possible to go back to previous steps of the workflow in order to change parameters and then proceed with the new result.

\subsection{Reconstruction}

We apply filtered back projection to reconstruct a 3D volume from the set of $2 \mathrm{D}$ projections. The filtered back projection by Feldkamp et al. (FDK) [4] is the reconstruction method which is widely used for cone-beam 3DXCT. The algorithm consists of two steps. First, a high-pass filter is applied to the projection images in order to eliminate blurring. The most commonly used high-pass filters are the ramp filter and the Shepp-Logan filter [27]. We use Shepp-Logan filtering in the reconstruction algorithm. Second, back projection is used to reconstruct the volume from the set of projection images. The basic idea of back projection is to sweep the projection images back through the $3 \mathrm{D}$ volume, accumulating intensity values at the voxels in $3 \mathrm{D}$ space. The back projection using unfiltered projection images would produce severely blurred images. The blurring effect appears because the frequency spectra of the projection images overlap in the low frequency region. Therefore, the prior high-pass filtering step is essential for reconstruction. The FDK reconstruction is comparatively fast and accurate which makes it most suitable for 3DXCT. However, it introduces some additional high-frequency noise to the volume due to the highpass pre-filtering. It also emphasizes metal streaking-artifacts when MMC specimens are reconstructed. As the FDK reconstruction performs an independent set of computations for every voxel, it is well suited for a GPU-based execution. In this work we use a filtered back projection implemented using the CUDA Toolkit [19]. The performance of the reconstruction benefits from the massive parallelization and computational power provided by the GPU. 

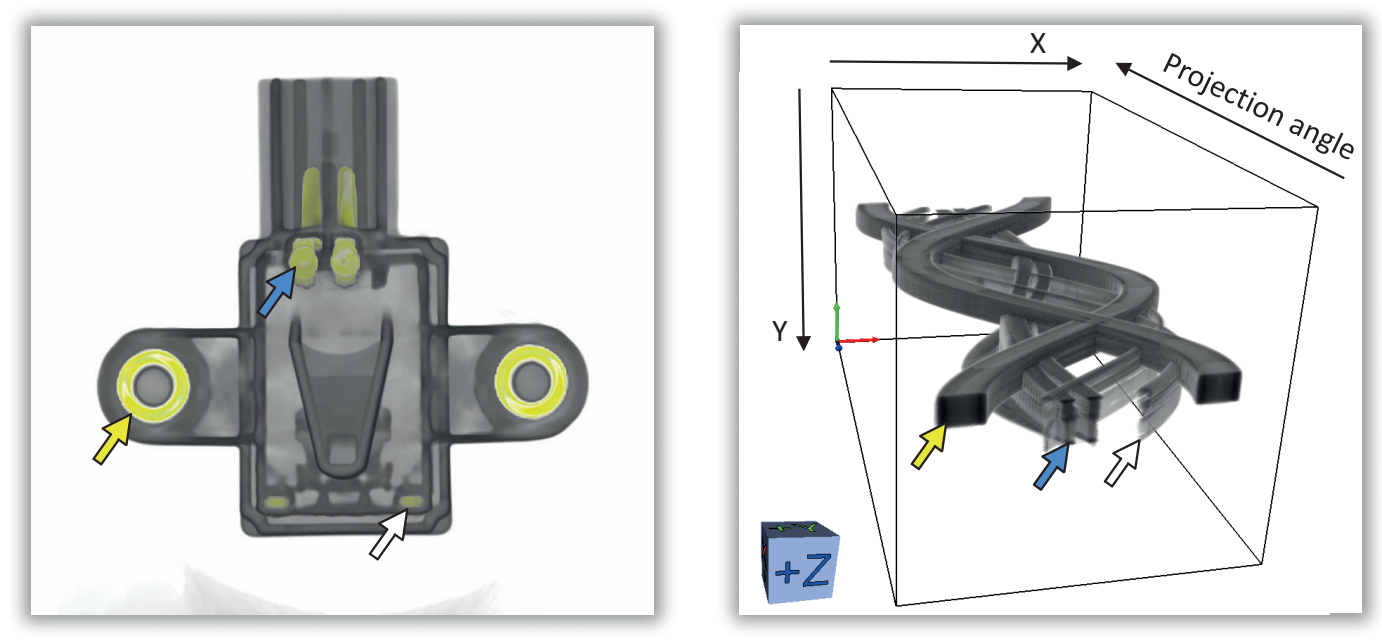

Fig. 6. Example of a 3D sinogram with trajectories of the metal parts of a specimen.

\subsection{Material Separation}

A reliable material separation is crucial for the final metal-artifact reduction. The segmentation of the metal components should include all the metal-affected areas of the projections. Furthermore, the segmentation should be accurate in order to avoid segmenting plastic or air parts where there is no presence of metal. MMCs containing small or thin metallic features (e.g., wires, thin pins, metal contacts or rivets) are the most difficult cases for material separation. It is not easy to distinguish such metal features from areas with thicker layers of plastic. Two material-separation approaches can be applied for the segmentation. The first approach is to perform the segmentation by directly operating on the set of $2 \mathrm{D}$ projections. Another approach is to compute the segmentation in the reconstructed $3 \mathrm{D}$ volume and then to perform a mapping of the resulting segmentation back to the $2 \mathrm{D}$ projections. Projection-based segmentation seems to be a better choice since the additional forward-projection step is not needed, but it has a set of major drawbacks. It is impossible to segment small metal elements as they produce the same attenuation of the X-rays as thick plastic components. Due to this, it is hard to properly segment the pixels partially affected by the metal. We have encountered the above problems during an attempt to develop an efficient projection-based segmentation technique. We have overcome the problem of segmenting partial-volume pixels by using a region-growing algorithm based on an initial segmentation. This gives a proper metal-artifact reduction on the simulated dataset with large metal pins. However this technique has failed on the real-world dataset with small metal elements. Therefore in this paper we use the $3 \mathrm{D}$ volume segmentation with a subsequent forward-projection step.

We segment metal using straight forward attenuation-coefficent thresholding in the reconstructed 3D volume. The threshold should be selected in a way to segment as much metal as possible, without touching any other materials. Subsequently, we forward-project the metal parts from the $3 \mathrm{D}$ volume to the $2 \mathrm{D}$ projections. This approach is helpful in difficult situations, if segmenting the metal in the projections is not immediately possible. Disadvantages of this approach are the higher computational costs and the precision errors. The first type of precision error is introduced by the reconstruction algorithm. During the discretization of the back projected image the reconstructed data is sampled into the volume grid. This leads to an information loss and restrains the exact inverse mapping using the forward projection. Another source of precision error is the imperfection of the forwardprojection algorithm.

\subsection{Forward Projection}

The goal of the forward-projection step is to project the segmented metal voxels from the reconstructed $3 \mathrm{D}$ volume back to the $2 \mathrm{D}$ projection images. The input to this workflow stage are the volume with segmented metal and the set of projection images. The areas of the projection images where metal is mapped to are filled with a void value. The output of the algorithm is a modified set of projection images. The forward projection is closely related to the reconstruction algorithm and uses the same transformations and scanning geometry.

An ideal forward projection would require solving a density integral for each projection pixel, where integration is done over the volume of the pyramid with apex at the X-ray source position and base at the detector cell. As exact forward projection is complex and computationally expensive, we use an approximate forward-projection algorithm.

The forward-projection works as follows:

- for every segmented voxel find the corresponding pixels on every projection image

- fill the corresponding pixels with the void value.

The forward-projection technique has to take into account partially covered pixels. Otherwise sampling artifacts may occur on the border of the projected metal. We include all the partially covered pixels to the projected metal area. For this purpose we calculate the length of the voxel diagonal mapped on the projection plane. This is the coverage diameter. Then we map the center of the voxel to the projection plane and find the corresponding pixel index. This is the center pixel. We consider all those pixels as covered which intersect with the axisaligned square positioned at the projected voxel center and with side length equal to the coverage diameter. This leads to an over-estimation of the projected metal area. The over-estimation should not be a serious problem because the non-metal density is later interpolated across the covered pixels. It would be more serious to miss removing any metal.

As forward-projection performs an independent sequence of computations for every voxel, it is well suited for a GPU-based execution. We used CUDA for the implementation. The parallelization is done in a way that every voxel is forward projected in a separate thread. The pseudocode of the forward-projection is listed in Algorithm 1.

\subsection{Interpolation}

The interpolation step of the workflow takes projections with voids as input and produces projections with filled voids as output. As voids are left in metal areas of the projections, we use interpolation to fill 


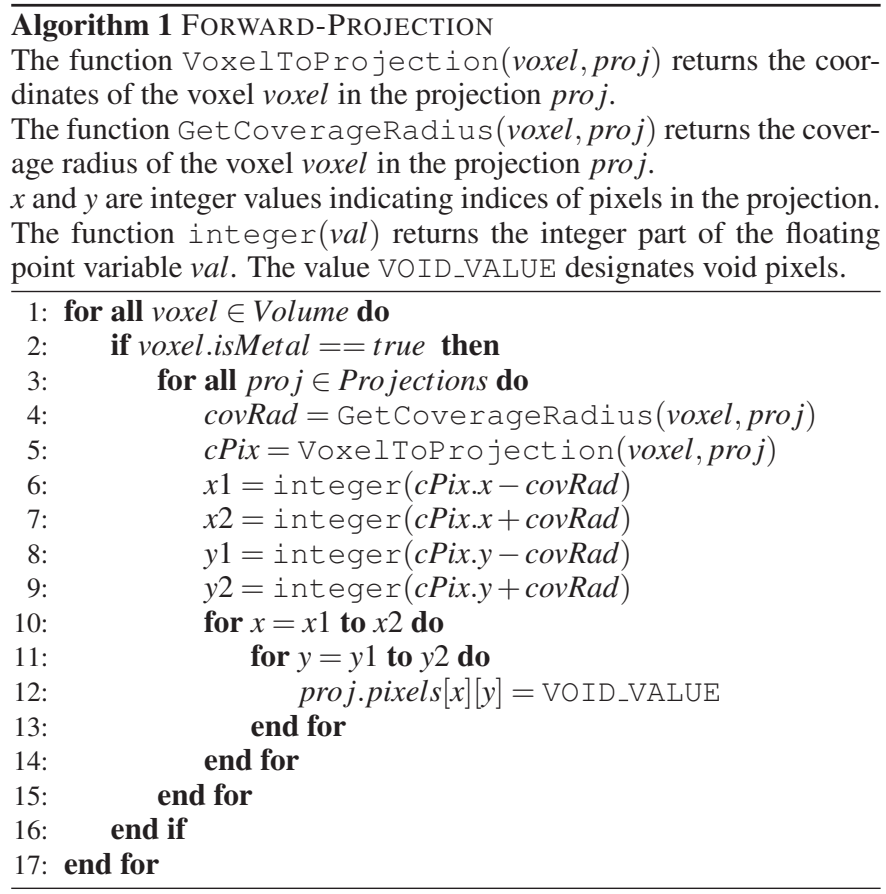

these empty regions. As approximation we use the information from the surrounding pixels. There are various ways to do the void interpolation. The interpolation can be done in $2 \mathrm{D}$ (taking into account only the data of a single projection image) or in 3D (taking into account the data of neighboring projections). Additionally, various interpolation techniques can be used. Examples are linear, bilinear, bicubic [16] or B-spline [29] interpolation.

Filling the voids is an image inpainting problem. Modern image inpainting methods mainly work with 2D images and are computationally expensive when applied to large 3D data. Therefore we consider a conventional interpolation strategy which is effective in processing large 3D volumes and which can be easily performed in parallel on the GPU. In this paper we use a 2D row-wise interpolation for regenerating the data in the voids. The interpolation works as follows:

- interpolation is done for every row of every projection image

- every pixel in the row is serially checked against the void value; the first and the last pixel of the void are detected

- when the last pixel of the void is reached, all pixels of the void are filled with interpolated values and the algorithm continues with the next pixel

- the row interpolation finishes when the last pixel of the row is reached.

In this work we do the interpolation only row-wise along the $\mathrm{X}$ axis as it is the optimal interpolation direction in many cases. However considering another interpolation direction based on the void geometry could improve the interpolation quality. The GPU implementation for the interplation is written in CUDA. Every row is interpolated in parallel within a separate thread. The pseudocode of the interpolation algorithm is listed in Algorithm 2.

\subsection{Fusion}

The fusion step of the workflow inserts the metal voxels from the volume containing segmented metal to the volume containing plastic with reduced metal artifacts. This is the $3 \mathrm{D}$ volume reconstructed from the projections containing plastic with interpolated voids. The result is a volume with reduced metal artifacts containing all the materials. We use the attenuation-coefficient threshold from the material-separation stage as a decision factor during the fusion.

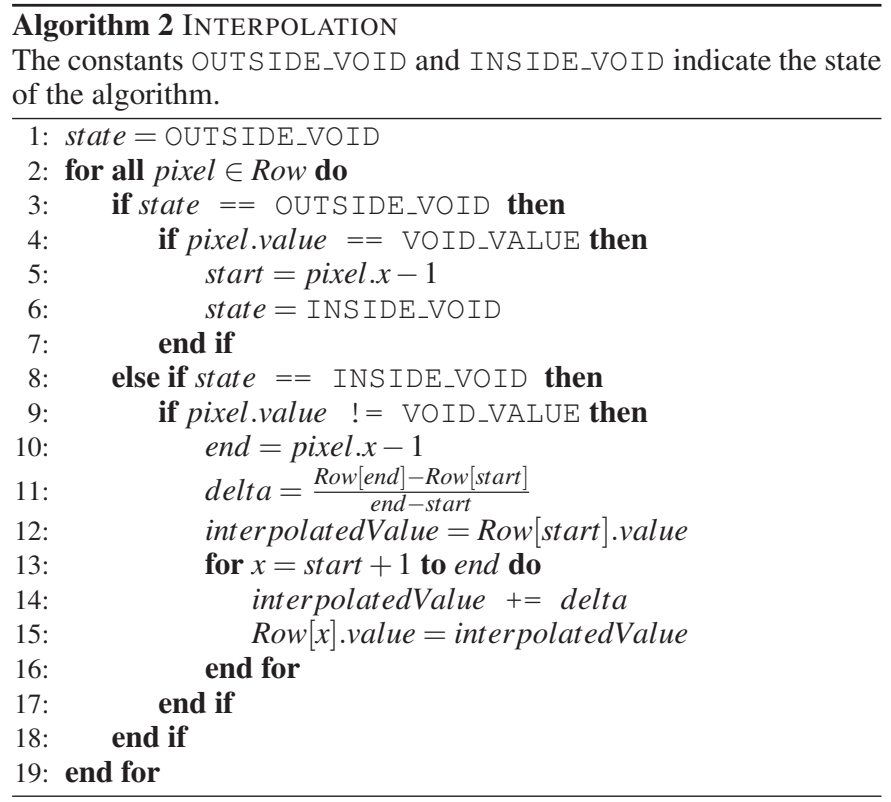

To achieve a smooth appearance at the borders of the metal elements we apply interpolation to the greyvalues within a certain range around the threshold. This range is controlled by the blend radius. We blend the voxel values which are within the blend radius from the threshold using linear interpolation. If the voxel value in the metal volume is higher than the threshold plus the blend radius, we use the voxel value of the metal volume. If the voxel value in the metal volume is lower than the threshold minus the blend radius, we use the voxel value of the MAR volume. The pseudocode of the Fusion algorithm is listed in Algorithm 3.

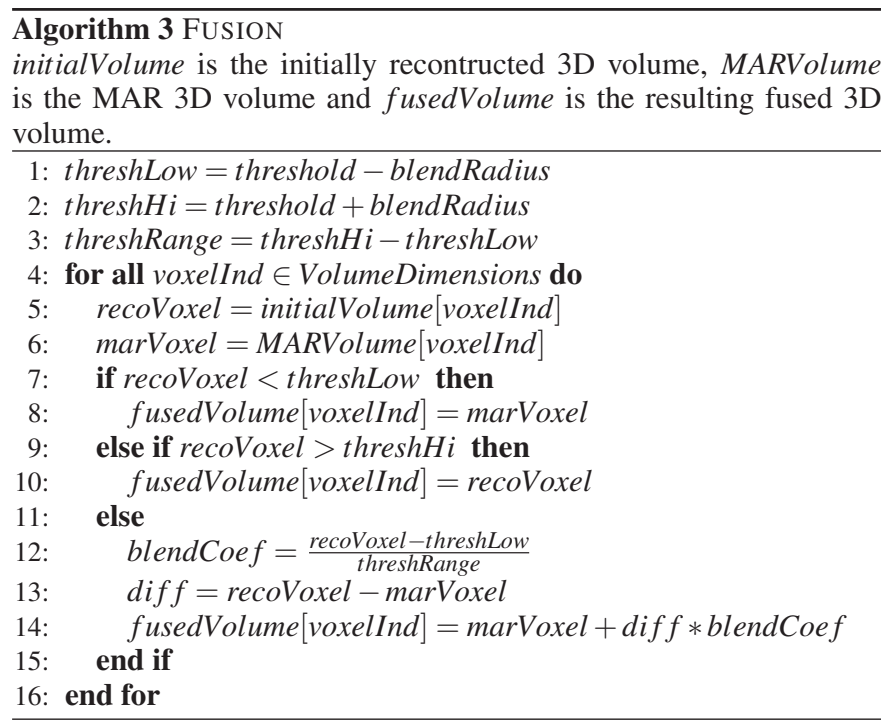

\section{Visual-Analysis tool for Metal-Artifact Reduc- TION}

We integrate all steps of the workflow in a visual-analysis tool. The basic idea is to provide the user with a convenient interface for executing the proposed MAR workflow on the various datasets. This is done by visually guiding the user through the steps of the workflow which require user input. User decisions are assisted with a set of visual-analysis techniques.

The main parts of the tool contain functionality concerning visual parameter estimation for the material separation (Section 4.1) and concerning the visual result exploration (Section 4.2). 
possible thresholds

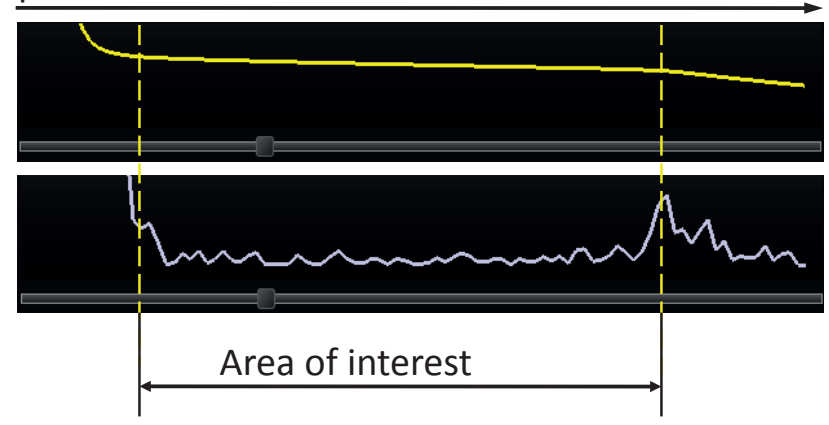

Fig. 7. The threshold-preview plot example for the cube specimen.

After the initial parameters are specified, the MAR workflow is executed for the selected projections by clicking the corresponding button. After the MAR workflow for the specified projections is successfully finished, the result will be displayed in a separate child window of the main application. This child window can be further used for various operations such as saving, exploring the data, volume rendering, or applying different filters.

\subsection{Visual Parameter Estimation for Material Separation}

The material-separation step of the workflow requires a threshold parameter to be specified by the user. The material-separation part of the tool helps the user to visually estimate the threshold, which is controlled using a slider. We provide the user with several visual tools allowing the estimation of the threshold. First, a threshold-preview plot can be constructed and shown above the slider. This widget plots the total volume of the segmented metal for the set of threshold values. This plot is visually linked with the slider so that the plot value gives the segmented metal volume for the threshold corresponding to this slider position. Using this plot the user can locate a threshold where the metal volume stops falling rapidly and reaches a stable size. This means that plastic is not included in the segmentation and no metal is false-negatively segmented. Instead of displaying the segmented metal volume, the absolute value of the second derivative thereof can be shown alternatively. An example of a threshold-preview plot is shown in Figure 7. The volume of the segmented metal is shown at the top and the second derivative of this function is depicted at the bottom of the image. The area where thresholding could provide good results is denoted.

Below the slider the slice view is located. This view shows a slice through the reconstructed volume. The slice direction, rotation and number can be specified using the corresponding GUI controls. The area showing the slice itself is fully interactive and allows zooming and shifting. Two additional widgets for the color map and the physical dimensions are shown on this view. When a voxel is covered by the mouse cursor, its index, position and data value are shown in the overlay box. The user can switch between the reconstructed 3D volume and the segmented 3D volume in the slice view. This allows an easy visual estimation of the parts in the reconstructed volume which are segmented for the given threshold value. A slice view is shown in Figure 8. Another helpful feature for the threshold estimation is the MAR-result preview. First, the user has to specify the range of the threshold to explore. Then the whole MAR workflow is executed for the thresholds in the given range. When the previews are calculated, the results are shown in the slice view. This conveys to the user how different thresholds directly affect the final result and allows selection of the threshold which provides the best MAR result. The user can switch the slice view between the resulting 3D volume and the difference between the resulting and the initial reconstructed 3D volumes. This information gives a good visual estimation of how the MAR workflow changed the initial volume.

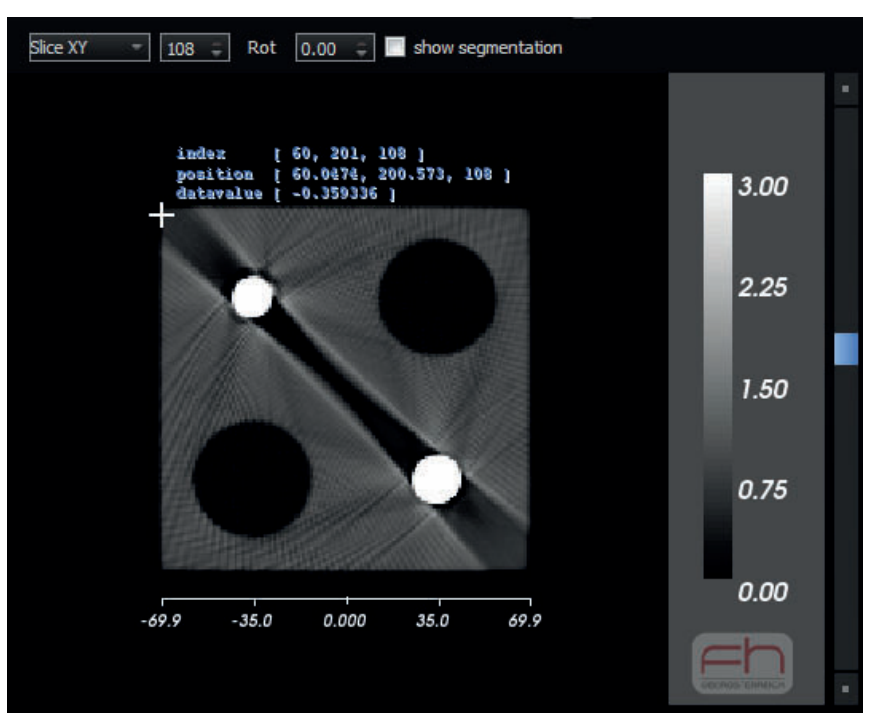

Fig. 8. A slice view showing the XY-slice of the CUBE specimen. Brightness settings of the image were enhanced for illustration purposes.

\subsection{Visual Result Exploration}

After all the parameters for the MAR workflow are set, the user can explore the difference between the initial reconstructed $3 \mathrm{D}$ volume and the MAR volume. He/she can smoothly move from the initial reconstruction to the MAR volume. The amount of blending is controlled by a slider. For the blending we use linear interpolation. The result is immediately shown in the slice view. Two transition steps from the initially reconstructed volume to the MAR volume are shown in Figure 9. Instant switching back and forth between the two images is available using the before/after buttons. It is clearly visible how metal artifacts represented by dark bands and streaking-noise are removed from the $3 \mathrm{D}$ volume. The tool is useful for the visual exploration of the artifact-reduction effect on the dataset. Changes in the dataset due to artifact reduction and the blurring introduced by the MAR workflow are visible using this interactive visual exploration tool.

\section{IMPLEMENTATION}

The prototype application was implemented in $\mathrm{C}++$. The slice view was implemented using VTK [24]. The GPU-based reconstruction, forward projection and interpolation were implemented using CUDA [19]. The data structures of ITK [12] were used for efficient handling of the $3 \mathrm{D}$ volume and the sinogram data. In addition, all the steps of the MAR workflow were implemented as ITK filters. This provides a nice abstraction from the voxel data type of the $3 \mathrm{D}$ volume and the pixel data type of the projection images. The ITK filter interface provides flexibile way of creating the filter pipelines by attaching the output of one filter to the input of another one.

\section{Results}

For the evaluation of the MAR workflow we use several specimens. The first test part was obtained using a simulation of a 3DXCT scan. For simulation we used the GPU-based 3DXCT simulation tool by Reiter et al. [22]. The simulated test part is a plastic cube with four drill holes: two larger ones and two smaller ones with steel pin insertions. We will further refer to this specimen as CUBE. The steel pins of the CUBE test part occupy a relatively big region in the dataset. Therefore, this specimen is well suited for testing the robustness of the workflow concerning the maximum size of metal features. Other test-parts are real-world multi-material components, obtained from a 3DXCT scan by using a PHOENIX X-RAY NANOTOM 180 scanning device. The first real-world specimen is a plug made of plastic with two big metal rivets on the sides and smaller metal contacts. We will further refer to this test part as PLUG1. Another real-world specimen is a plug as 

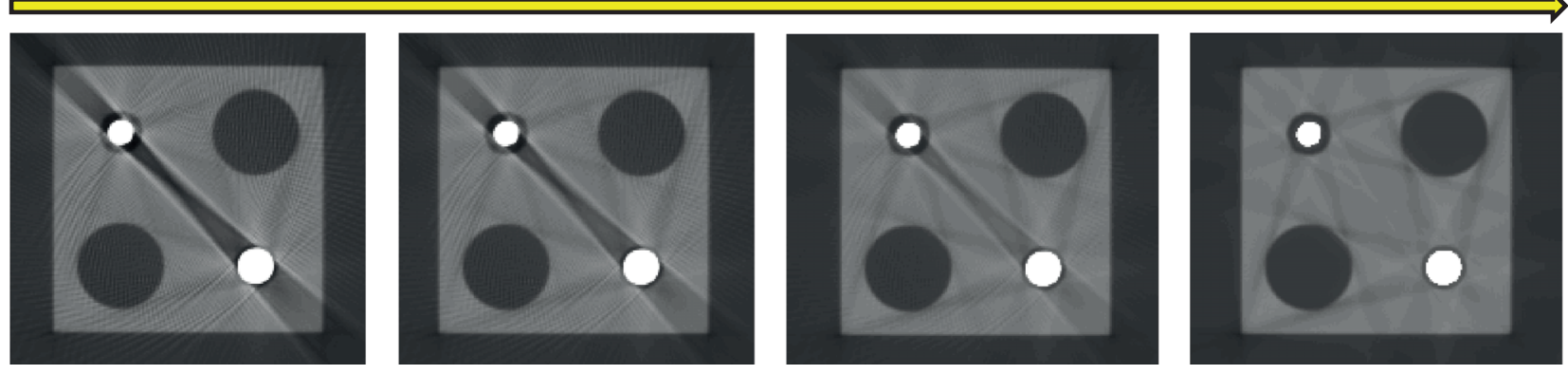

Fig. 9. Exploration of the MAR result. Smooth transition between the initial reconstruction and the MAR result is shown. The numbers on the top show the blending between the initial reconstruction and the MAR volume. Brightness and contrast settings of the images were enhanced for the illustrative purposes.

well. The carrier made of plastic contains several small metal contacts. In addition to the 19 contact elements, this specimen has three largerscale rivets located on the sides. We will further refer to this test part as PLUG2. The scanning presets used for the PLUG1 test part are: voltage $=170 \mathrm{kV}$, current $=400 \mu \mathrm{A}$. The scanning modalities for the PLUG2 specimen are: voltage $=175 \mathrm{kV}$, current $=400 \mu \mathrm{A}$. For both test parts 900 projections were taken with an integration time of 1000 $m s$ per projection. The projection images were downsampled from $1024 \times 1024$ to $256 \times 256$ pixels using a bicubic interpolation method. The downsampling was done for testing purposes; the data resolution does not limit our method. Due to the small size of the metal contacts, the PLUG1 and the PLUG2 specimens are well suited for testing the robustness of the workflow with respect to the large number of small metal features. All datasets used in the MAR evaluation have severe metal artifacts distorting the plastic and air regions and making a reliable material characterization impossible.

The results of applying the proposed workflow to the CUBE, PLUG1, and PLUG2 test parts are given in Figures 10, 11, and 12, respectively. The images on the left side show the original reconstructed 3D volumes, while the images on the right side depict the corrected 3D volumes. In all cases the improvement in volume and slice quality is clearly visible. By applying the proposed metal-artifact reduction workflow we managed to significantly improve the result for all specimens. The material interfaces got sharper and the amount of incorrectly classified plastic was significantly reduced. This allows us to achieve a higher quality material characterization and perform the material analysis in areas where it was not possible before. On the other hand, the metal appearance is preserved. The greyvalues of the metal correspond to the values in the original reconstructed volume. Sometimes the MAR method introduces blurring of the plastic edges due to interpolation. The amount of this blurring depends on the geometry of the specimen conveyed by the area and the location of the metal parts.

Our approach does not treat beam-hardening artifacts introduced by the plastic. Only metal artifacts are corrected. Suppression of the streaking artifacts results in a lower noise-level in the dataset. This leads to a more evident appearance of the plastic beam-hardening artifacts. These artifacts are present in the original data as well and MAR does not amplify them. This may be one of the reasons for the starshaped artifacts surrounding the metal parts of the CUBE specimen. The residual artifacts may as well arise from metal parts (due to under segmentation), or from other unconsidered effects.

\section{Conclusion}

In this paper we adapted a projection-based metal-artifact reduction workflow for 3DXCT and in particular for industrial 3DXCT. We present a visual tool integrating all steps of the described MAR workflow. The tool guides the user through the set of steps. It provides visual-analysis functionality allowing interactive parameter estimation for the material separation stage. Additionally, the visual exploration of the MAR result and of the workflow impact on the volume is available. We have evaluated the presented workflow on both simulated and real-world MMC specimens with metal substructures of various sizes. Metal-artifact reduction was achieved for all tested MMCs. The visual evaluation shows the significant reduction of the metal artifacts. The artifact reduction enables a reliable dimensional measurement and material exploration in areas where this was not possible without scans of the disassembled specimen. This helps industrial 3DXCT experts to save scanning time and resources.

The $3 \mathrm{D}$ sinogram allows taking into account neighboring projections. Therefore additional information can be used in the materialseparation and the void-interpolation stages of the MAR workflow. In the presented algorithm we do not make use of such additional information. Using interpolation schemes that operate in 3D space and improving the segmentation in the reconstructed $3 \mathrm{D}$ volume by checking against the 3D sinogram are promising topics for further research. The quality of the metal segmentation is essential for achieving a good MAR result. Our material separation requires the user to define the threshold parameter. One important assumption of this segmentation technique is that the scanned data allows metal separation by thresholding. This requires that the density difference of the metal and other materials of the object is large enough to allow a proper distinction. In problematic cases (e.g., industrial MMC composed of many materials with close densities) more sophisticated segmentation techniques need to be applied. Another limitation is that the error estimation of the MAR workflow is difficult, as in most cases the ground truth is not available. In future work an error estimation could be performed by comparing the MAR results with artifact-free simulations or scans of the disassembled specimen. Additionally, applying the workflow to dual-energy 3DXCT could improve the quality of metal-artifact reduction. Despite the mentioned limitations, the presented MAR algorithm shows a significant metal-artifact improvement for the industrial MMCs containing metal and plastic and is quite advantageous for further material characterization and exploration.

\section{ACKNOWLEDGMENTS}

The presented work has been funded by the Bridge-Project SmartCT and the K-Project ZPT (http://www.3dct.at) of the Austrian Research Promotion Agency (FFG). Thanks to the vis-group of the Vienna University of Technology, Institute of Computer Graphics and Algorithms, for support in designing this method and the CT group of the Upper Austrian University of Applied Sciences - Wels Campus for CT measurements, reference measurements and illustrations. We also thank Marius Gavrilescu, for his comments on the manuscript on a short notice. 

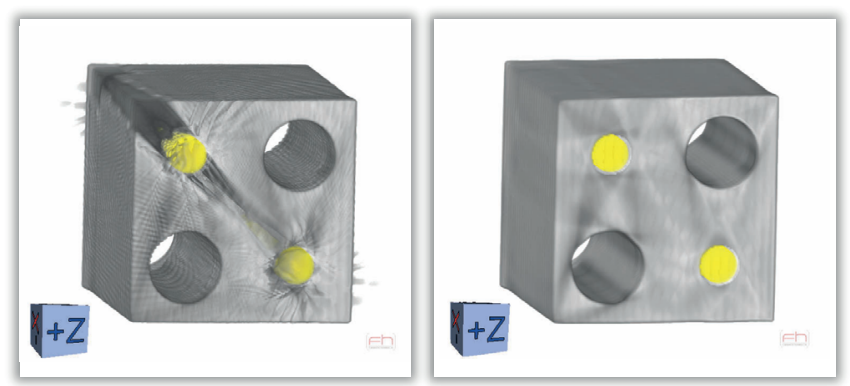

(a)
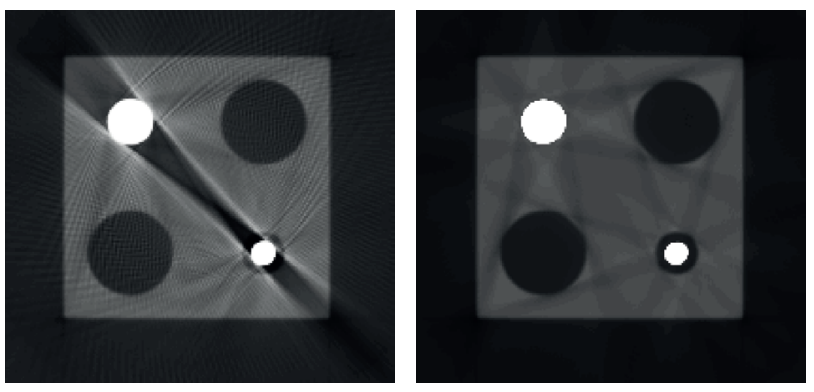

(b)

Fig. 10. The results of the metal-artifact-reduction workflow for the simulated CUBE specimen. On the left the initial dataset is shown, on the right the same dataset after the metal-artifact-reduction workflow is given. Volumetric rendering (a) and XY-slice image (b) comparisons are given.
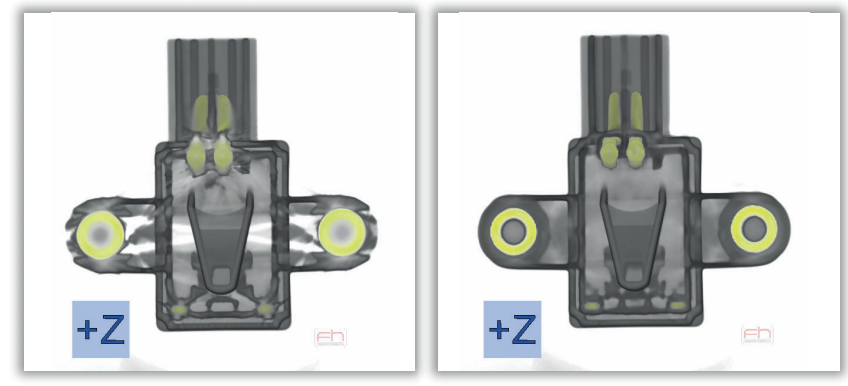

(a)
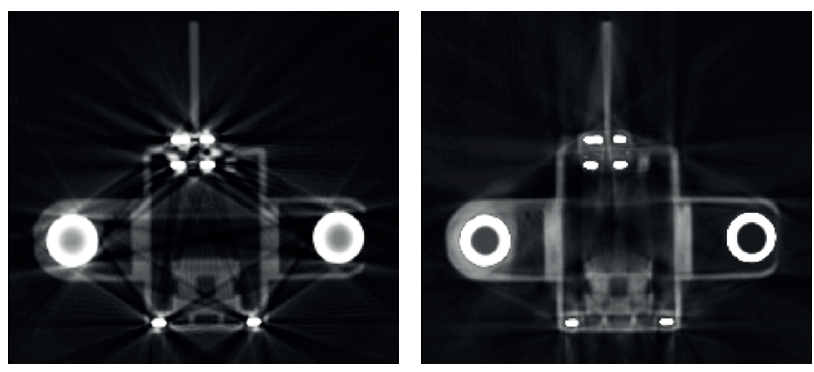

(b)

Fig. 11. The results of the metal-artifact-reduction workflow for the realworld PLUG1 specimen. On the left the initial dataset is shown, on the right the same dataset after the metal-artifact-reduction workflow is given. Volumetric rendering (a) and XY-slice image (b) comparisons are given.
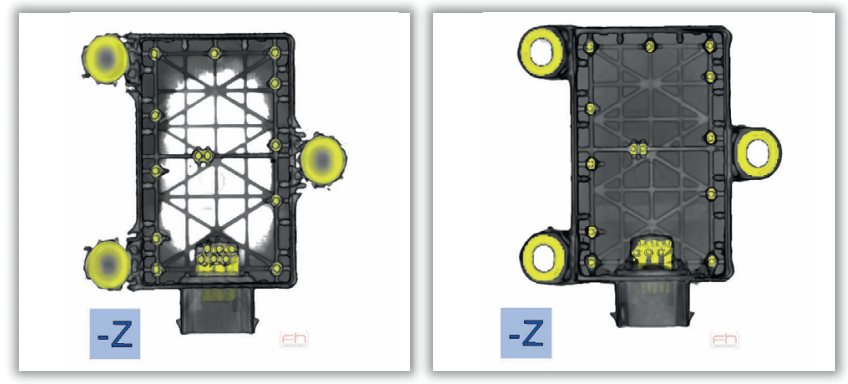

(a)
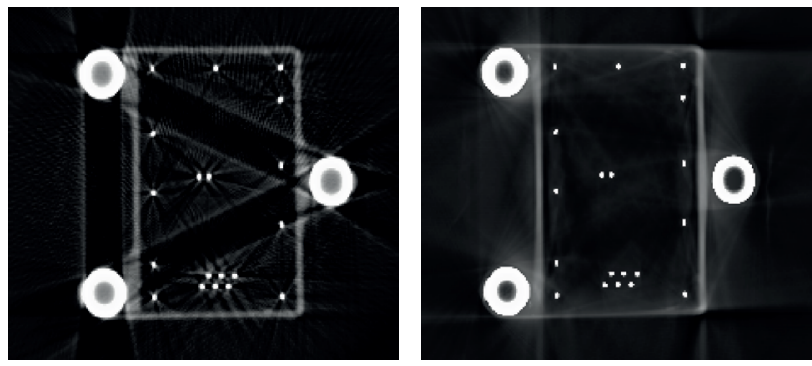

(b)

Fig. 12. The results of the metal-artifact-reduction workflow for the realworld PLUG2 specimen. On the left the initial dataset is shown, on the right the same dataset after the metal-artifact-reduction workflow is given. Volumetric rendering (a) and XY-slice image (b) comparisons are given. 


\section{REFERENCES}

[1] J. F. Barrett and K. Nicholas. Artifacts in CT: Recognition and avoidance. Radiographics ISSN 0271-5333, vol. 24(6):1679-1691, 112004.

[2] T. F. Chan and J. Shen. Mathematical models for local nontexture inpaintings. SIAM J. Appl. Math, 62:1019-1043, 2002.

[3] X. Duan, L. Zhang, Y. Xiao, J. Cheng, Z. Chen, and Y. Xing. Metal artifact reduction in CT images by sinogram TV inpainting. In Nuclear Science Symposium Conference Record, 2008. NSS '08. IEEE, pages 4175 4177, 102008.

[4] L. A. Feldkamp, L. C. Davis, and J. W. Kress. Practical cone-beam algorithm. J. Opt. Soc. Am. A, 1(6):612-619, 61984.

[5] M. Hadwiger, F. Laura, C. Rezk-Salama, T. Hollt, G. Geier, and T. Pabel. Interactive Volume Exploration for Feature Detection and Quantification in Industrial CT Data. IEEE Transactions on Visualization and Computer Graphics, 14(6):1507 -1514, 11 - 122008.

[6] C. Heinzl. Analysis and Visualization of Industrial CT Data. $\mathrm{PhD}$ thesis, Vienna University of Technology, 122009.

[7] C. Heinzl, J. Kastner, T. Möller, and M. E. Gröller. Statistical Analysis of Multi-Material Components using Dual Energy CT. In D. S. Oliver Deussen, Daniel Keim, editor, VMV 2008, Vision, Modeling and Visualization, pages 179-188, 102008.

[8] G. T. Herman. Correction for beam hardening in computed tomography. Physics in Medicine and Biology, 24(1):81, 1979.

[9] F. Hopkins, Y. Du, B. Lasyuk, A. Abraham, and S. Basu. Analytical corrections for beam-hardening and object scatter in volumetric computed tomography systems. In WCNDT, volume 22, pages 462-468, 2004.

[10] J. Hsieh. Computed Tomography: Principles, Design, Artifacts and Recent Advances. SPIE Press, 22003.

[11] R. Huang, K.-L. Ma, P. McCormick, and W. Ward. Visualizing Industrial CT Volume Data for Nondestructive Testing Applications. Visualization Conference, IEEE, page 72, 2003.

[12] L. Ibanez, W. Schroeder, L. Ng, and J. Cates. The ITK Software Guide. Kitware, Inc. ISBN 1-930934-15-7, http://www.itk.org/ItkSoftwareGuide.pdf, second edition, 2005.

[13] G.-R. Jaenisch, C. Bellon, U. Samadurau, M. Zhukovskiy, and S. Podoliako. A Monte Carlo Model Coupled to CAD for Radiation Techniques. European Conference on Non Destructive Testing (CD-ROM), 2006.

[14] W. A. Kalender, R. Hebel, and J. Ebersberger. Reduction of CT artifacts caused by metallic implants. Radiology, 164(2):576-7, 1987.

[15] S. Kasperl. Qualitätsverbesserungen durch referenzfreie Artefaktreduzierung und Oberfächennormierung in der industriellen $3 D$ Computertomographie. $\mathrm{PhD}$ thesis, Technische Fakultät der Universität Erlangen Nürnberg, 2005.

[16] R. Keys. Cubic convolution interpolation for digital image processing. IEEE Transactions on Acoustics, Speech and Signal Processing, 29(6):1153 - 1160, 121981.

[17] M. Krumm, S. Kasperl, and M. Franz. Reducing non-linear artifacts of multi-material objects in industrial 3D computed tomography. $N D T \& E$ International, 41(4):242 - 251, 2008

[18] N. Freud and J.-M. Létang and D. Babot. A hybrid approach to simulate multiple photon scattering in X-ray imaging. Nuclear Instruments and Methods in Physics Research, pages 551-558, 2005.

[19] NVIDIA. CUDA Programming Guide 2.3, 2009.

[20] M. Oehler and T. M. Buzug. Sinogram Inpainting for Metal Artifact Reduction in CT Images. In Proc. 4th European Congress for Medical and Biomedical Engineering, Springer IFMBE Series, volume 22, pages 651-654, 2008.

[21] V. Rebuffel and J.-M. Dinten. Dual-Energy X-Ray Imaging: Benefits and Limits. In European Conference on Non Destructive Testing (CD-ROM), 2006.

[22] M. Reiter, M. M. Malik, C. Heinzl, D. Salaberger, M. E. Gröller, H. Lettenbauer, and J. Kastner. Improvement of X-Ray image acquisition using a GPU based 3DCT simulation tool. In 8th International Conference on Quality Control by Artificial Vision, page 8, 52009.

[23] M. Reiter, M. Mantler, and B. Chyba. McRay - A Monte Carlo Simulation of Projections in Computed Tomography. Denver X-ray Conference 2007, Denver (US), 7-8 2007

[24] W. Schroeder, K. Martin, and B. Lorensen. The Visualization Toolkit, Third Edition. Kitware Inc., 2007.

[25] X. Shao, Z. Liu, and H. Li. An Image Inpainting Approach Based on the Poisson Equation. In Proceedings of the Second International Confer- ence on Document Image Analysis for Libraries, pages 368-372. IEEE Computer Society, 2006.

[26] J. Shen and T. F. Chan. Mathematical models for local nontexture inpaintings. SIAM Journal on Applied Mathematics, 62(3):1019 - 1043, 12 2002.

[27] L. A. Shepp and B. F. Logan. The Fourier Reconstruction of a head section. IEEE Transactions on Nuclear Science, NS-21(3):21-43, 1974.

[28] J. Tabary, R. Guillemaud, F. Mathy, A. Gliére, and P. Hugonnard. Combination of high resolution analytically computed uncollided flux images with low resolution Monte-Carlo computed scattered flux images. Proc. IEEE-MIC, Norfolk, pages 551-558, 112002.

[29] P. Thévenaz, T. Blu, and M. Unser. Interpolation revisited. IEEE Transactions on Medical Imaging, 19(7):739-758, 72000.

[30] W. J. H. Veldkamp, R. M. S. Joemai, A. J. van der Molen, and J. Geleijns. Development and validation of segmentation and interpolation techniques in sinograms for metal artifact suppression in CT. Medical Physics, 37(2):620-8, 2010

[31] F. Xu and K. Mueller. Ultra-Fast 3D Filtered Backprojection on Commodity Graphics Hardware. IEEE International Symposium on Biomedical Imaging (ISBI '04), pages 571-574, 42004.

[32] F. Xu and K. Mueller. Real-time 3d computed tomographic reconstruction using commodity graphics hardware. Physics in Medicine and Biology, 52:3405-3419, 2007.

[33] H. Xue, L. Zhang, Y. Xiao, Z. Chen, and Y. Xing. Metal artifact reduction in dual energy CT by sinogram segmentation based on active contour model and TV inpainting. In Nuclear Science Symposium Conference Record (NSS/MIC), 2009 IEEE, pages 904 -908, 112009.

[34] L. Yu, J. Kofler, X. Liu, A. Primak, and C. McCollough. Automatic Metal Artifact Reduction From Reformatted Projections in Multi-Slice Helical CT. Medical Physics, 35:2949, 2008. 